\title{
Anthropometric Predictors and Artificial Neural Networks in the diagnosis of Hypertension
}

\author{
Krzysztof Pytel \\ University of Lodz, \\ Faculty of Physics and \\ Applied Informatics, \\ Lodz, Poland \\ Email: kpytel@uni.lodz.pl
}

\author{
Tadeusz Nawarycz, \\ Lidia Ostrowska-Nawarycz \\ Medical University of Lodz, \\ Department of Biophysics, \\ Lodz, Poland
}

\author{
Wojciech Drygas \\ Medical University of Lodz, \\ Department of Epidemiology, \\ Lodz, Poland
}

\begin{abstract}
Artificial Neural Networks (ANNs) play a vital role in the medical field in solving various health problems like estimating the risk of cardiovascular diseases. The article concerns the process of developing ANNs for estimating the risk of arterial hypertension. ANNs proposed in this article use anthropometrical predictors, easy to control for everybody at home without special equipment. In the article we analyze four different models of ANNs and try to find out which model and set of anthropometrical predictors estimates the risk the most accurately. We use dataset of 2485 real cases of patients from the city of Lodz. The experiment was done in the Matlab environment. The performance of the proposed method in terms of accuracy and facility of use shows that ANNs can be effective tools for preliminary tests of arterial hypertension.
\end{abstract}

\section{INTRODUCTION}

A RTERIAL hypertension (HT) as a major risk factor for the development of cardiovascular diseases (CVD) constitutes an important problem of public health in Poland and other countries around the world [1]. Arterial HT is a disease that affects a wide range of the population, particularly the elderly after the age of 55. In compliance with the data given by multicenter Polish population health status studyWOBASZ, arterial hypertension appears among about $42.1 \%$ of men and $32.9 \%$ of women in Poland [2].

The diagnostics of HT as a sickness is based on:

a) the showing of increased values of blood pressure (based on their repeated measurement),

b) the estimation of the degree of the disease,

c) the differentiating of its etiology and results.

The value $140 \mathrm{mmHg}$ for systolic blood pressure (SBP) and/or $90 \mathrm{mmHg}$ the diastolic blood pressure (DBP) have been accepted for grown-ups as a border-line of hypertension [3]. The same classification is used for young, middle-aged and elderly subjects, whereas different criteria, based on percentilage for boys and girls according to their age and height [4] The state of high risk of development of HT, the so-called pre-hypertension state (PHT), is defined when SBP amounts 120 - $139 \mathrm{mmHg}$ and/or DBP amounts $80-89 \mathrm{mmHg}$. The patient with PHT needs the estimation of other cardiovascular risk and factors and modification of the lifestyle in the first instance.
Overweight and obesity are the most widespread occurrent environmental factor, that can cause the development of arterial hypertension (HT). The increased prevalence of obesity (especially visceral obesity) and other cardiovascular risk factors are closely associated with the rising incidence of CVD and type 2 diabetes mellitus. The enlarged quantity of the adipose tissue ties in with hyperinsulinemia and insulin resistance, which contributes to the level of blood pressure. Hormones and cytokines produced in adipocytes (eg. leptin, resistin, adiponektion, the interleukin-6, tumor necrosis factoralpha and others) play a key role, and emitted to the circulation of blood system, regulate lipid and glucose metabolism [5]. In Framingham research $70 \%$ of men and $60 \%$ of women with HT and with coexisting overweight or obesity, had their systaltic blood pressure increased about $4.5 \mathrm{mmHg}$ on every $5 \mathrm{~kg}$ of overweight [6].

Similar relations between the Body Mass Index (BMI) and arterial blood pressure were shown also in INTERSALT research [7]. With people having the similar body height, a 10 $\mathrm{kg}$ difference caused the increase of systaltic/diastolic pressure by $3 / 2.2 \mathrm{mmHg}$ respectively.

In Poland, the result of the IDEA (The International Day for Evaluation of Abdominal Obesity) research on grown-ups aged from 18 to 80, showed that the frequency of appearing of both abdominal overweight and arterial hypertension is one of the highest in Europe [8].

Considerably more often than among our European neighbours, both from the North and West, one ascertained abdominal overweight (in Poland: 54\% of women and 38\% of men; in north-west Europe: $45 \%$ of women and $33 \%$ of men). Besides, it was observed that arterial hypertension more often appears among people with overweight or obesity when compared to their slim peers of the same age. Stout people more often suffered from diseases of the cardiovascular (CVD) system, diabetes and dyslipidemia. Very often these diseases coexisted with overweight or obesity. The investigation the IDEA concluded that both among men and women, abdominal obesity and the BMI tie in with CVD independently.

In spite, of the well-known correlation between HT and overweight, controversies about which anthropometrical factors (general and abdominal overweight and other such pa- 
rameters as age and sex determine the most effective model of HT prediction) exist all the time.

In our work, we used ANNs, and the data from the research adults aged from 20 to 80 from the city Lodz, and we executed estimations of the efficiency of HT diagnostic for different models and anthropometrical data.

\section{DAtaset for Artificial Neural Networks}

The Artificial Neural Networks (ANNs) are constructed using various predictors, to be trained, tested and validated using the respective data sets. In experiments we used the set of real data concerning patients from the city of Lodz, aged from 20 to 80 . The set of data embraced 2485 cases of patients, 1197 men and 1288 women. Patients who did not have hypertension is the largest group (1370 cases, whereof 624 is men and 746 is women). In the group of patients with the 1-st stage of hypertension, there were 569 cases (315 men and 254 women). The group of patients with the 2-nd stage hypertension embraced 464 cases (221 men and 243 women). The least numerous group of patients was the one with the 3 -rd stage of hypertension and if consisted of 82 cases (37 men and 45 women).

The dataset was divided into a training set and a test set. The training set was used for neural network training, that is for adjustment of weights between neurons. The test set was used after the network was trained, to test ANNs accuracy on new data (not used for training).

\section{Models of the Artificial Neural Networks}

Artificial Neural Networks (ANNs) are algorithms inspired by function of the human brain. ANNs are usually presented as systems of interconnected "neurons" divided into a few layers. Each neuron can compute values from inputs and is capable of machine learning. Each neuron has a certain number of inputs, a real number associated with each connection (the weight of the connection), and has its own transfer function. The behaviour of an ANN depends on the weights and the transfer function. Most of the algorithms used in training ANN employ some form of backpropagation method. In supervised learning, a given set of input/output pairs is presented to the network. A learning algorithm tries to adjust connection weights to minimize the average squared error between the network's output and the target value for all the example pairs. ANNs can produce the values between 0 and 1 for any input vector. The advantage of ANNs is their ability to learn from the observed data. They can be used to infer a function from observations. This is useful in complex applications or tasks, where discovering such a function by hand is impractical or impossible.

All the experiments were done in the Matlab environment. In the experiments we used three-layer artificial neural networks. The number of neurons in input, hidden and output layers was fixed to 50,30 and 1 respectively. The proposed structure of the network was obtained as a result of a series of initial tests during which we were testing different structures. The hyperbolic tangent sigmoid transfer function (tansig) was

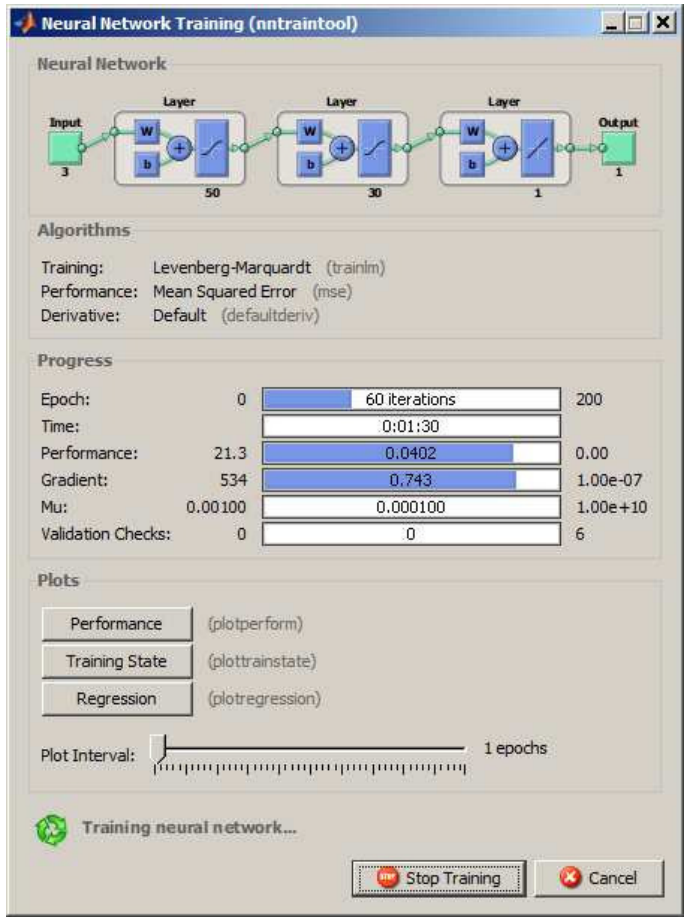

Fig. 1. An example of a three-layer artificial neural network teaching with the Levenberg-Marquardt method

used in the input and hidden layers, and the linear function (purelin) in the output layer. A Levenberg-Marquardt method with 200 cycles was used for teaching the networks. In Fig. 1 we show an example of the network teaching in the Matlab environment.

The result of the classification was represented on the output layer in the form of a real number. We accepted that output values from 0 to 0.17 represent the lack of hypertension, values from 0.17 to 0.5 represent the 1 st degree hypertension, values from 0.5 to 0.83 represent 2 nd degree hypertension, and values above 0.83 represent the 3 rd degree hypertension. In experiments we tested four models of the network with different input values:

- model 1: we accepted 3 inputs: the Body Mass Index (BMI), the Waist Circumference (WC) and the Age. On the output of the network we received the real number representing the result of the classification;

- model 2: we accepted 3 inputs: the Body Mass Index (BMI), the Waist Circumference normalized in relation to the BMI (WCBMI) and the Age. On the output of the network we received the real number representing the result of the classification;

- model 3: we accepted 4 inputs: the Body Mass Index (BMI), the Waist Circumference (WC), the Sex and the Age. On the output of the network we received the real number representing the result of the classification;

- model 4: we accepted 4 inputs: the Body Mass Index (BMI), the Waist Circumference normalized in relation to the BMI (WCBMI), the Sex and the Age. On the output 


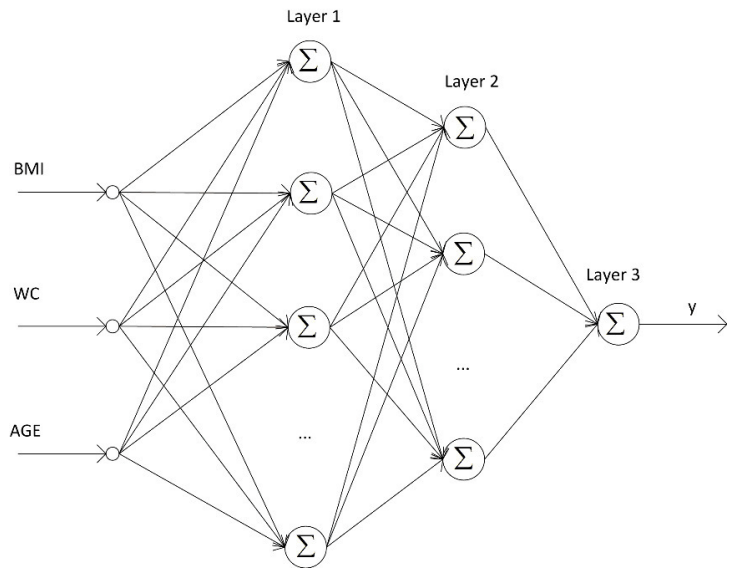

Fig. 2. Artificial Neural Network Architecture

TABLE I

THE RESUlTS OBTAINED BY EACH MODEL OF NEURAL NETWORK (THE AVERAGE NUMBER OF INCORRECTLY RECOGNIZED CASES IN PERCENTAGES)

\begin{tabular}{|c|c|c|c|c|}
\hline & HT 0 & HT 1 & HT 2 & HT 3 \\
\hline model 1 & 7.96 & 10.42 & 17.76 & 21.18 \\
\hline model 2 & 6.68 & 11.94 & 16.6 & 17.3 \\
\hline model 3 & 6.52 & 9.82 & 15.18 & 14.86 \\
\hline model 4 & 7.00 & 11.92 & 16.9 & 19.92 \\
\hline
\end{tabular}

of the network we received the real number representing the result of the classification.

In models 3 and 4, a non-numerical attribute (Sex) was used. The value of this attribute was converted into number: 0 for women and 1 for men.

In Fig. 2 we present the Architecture of Artificial Neural Network.

\section{EXPERIMENTAL RESULTS}

For the test we used all four proposed models of ANNs. Every network was run five times, embracing the learning process, and then, the test of the efficiency of the classification. The obtained result is the average from all five runs. The results obtained by each neural network are collected in table 1. The values in the table are in percent ages and represent the average number of incorrectly recognized cases with the lack of hypertension (HT0), hypertension of the first stage (HT1), hypertension of the second stage (HT2) and hypertension of the third stage (HT3). During the experiments, we used only the results of anthropometrical measurement and systaltic (SBP) and diastolic (DBP) blood pressure. The other factors, such as medicines, family conditionings and other, were not taken into account.

The comparison of model 1 and model 2, let on to check which input value (WC or WCBMI) better determines the results of the classification. The graph in Fig. 3 illustrat the differences in the average number of incorrectly diagnosed cases by neural networks with the use of models 1 and 2 .
The average number of incorrectly diagnosed cases

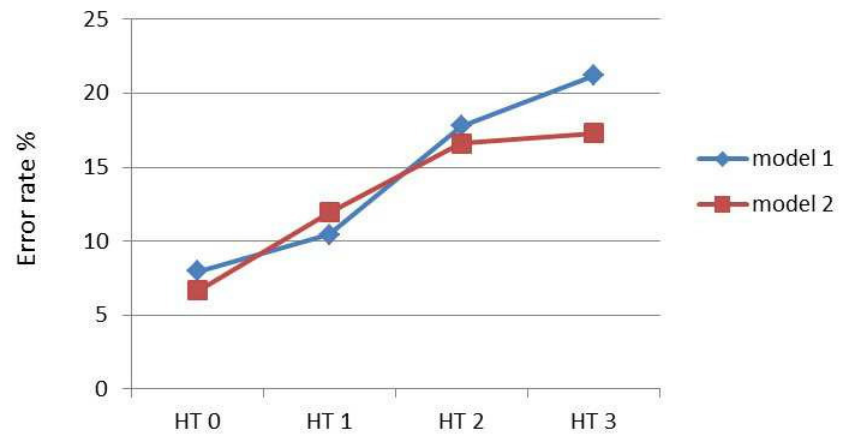

Fig. 3. Differences in the average number of incorrectly diagnosed cases by neural networks with the use of models 1 and 2 .

\section{The average number of incorrectly diagnosed cases}

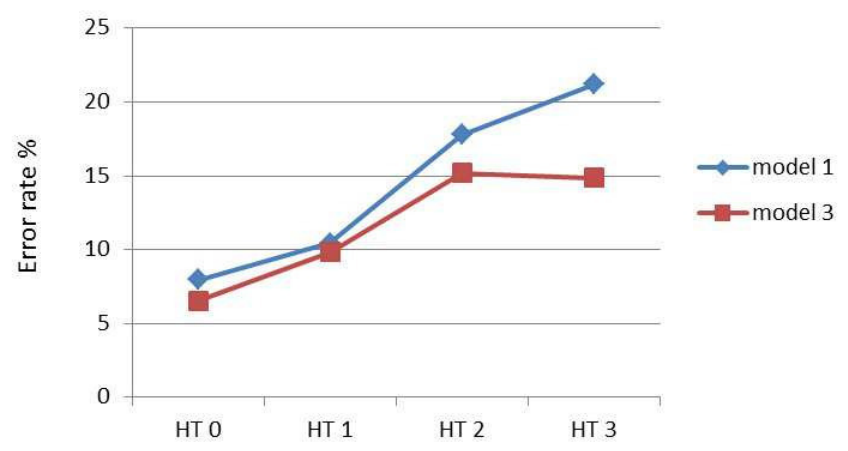

Fig. 4. Differences in the average number of incorrectly diagnosed cases by neural networks with the use of models 1 and 3 .

The graph in Fig. 4 illustrates differences in the average number of incorrectly diagnosed cases by neural networks with the use of models 1 and 3 .

Models 3 and 4 let on to check if the additional parameter (Sex) will improve exactitude of the classification and how it influences the value of the hypertension. The graph in Fig. 5 illustrates the average number of incorrectly diagnosed cases by neural networks with the use of all four models.

\section{CONCLUSIONS}

Overweight and obesity leading to arterial hypertension are a serious problem of the population of Poland and other countries around the world. Artificial Neural Networks, basing on anthropometrical factors, which are easy to measure at home, let on to make diagnostic reconnaissances towards the risk of arterial hypertension.

The neural network in which we used the Waist Circumference normalized in relation to the BMI (WCBMI), as the input (model 1) obtained a greater accuracy than the network in which we used the Waist Circumference without normalization (model 2). 


\section{The average number of incorrectly diagnosed cases}

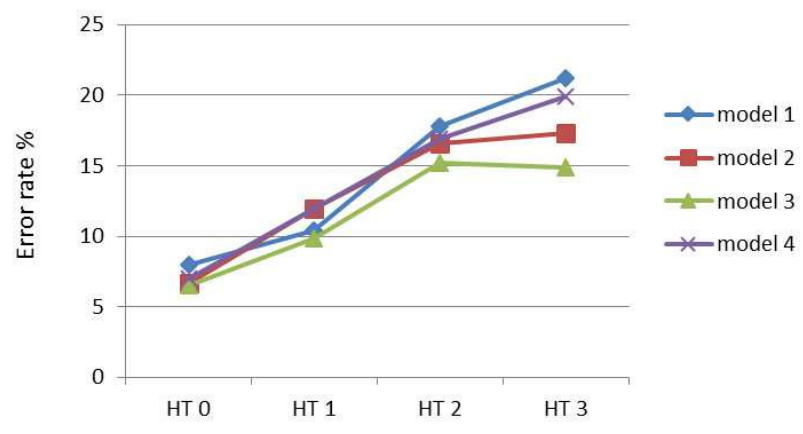

Fig. 5. Average number of incorrectly diagnosed cases by neural networks with the use of all four models.

The introduction of the additional input parameter to the network (the Sex), brought about the enlargement of the accuracy of the network. The greatest difference can be observed in the third stage of hypertension, though in the test set in this category the least cases appeared.

The results of our study may be a starting point for the construction of a more complex ANN system for the assessment of global arterial hypertension risk. Taking into account the growing scale of overweight and obesity epidemic around the world, the Neural Networks systems should be more widely used for diagnosis of this problem.

\section{REFERENCES}

[1] Hajjar I, Kotchen JM, Kotchen TA. Hypertension: trends in prevalence, incidence, and control. Annu Rev Public Health. 2006;27:465-90.
[2] Tykarski A., Posadzy-Malaczynska A., Wyrzykowski B. i wsp.: Rozpowszechnienie nadcisnienia tetniczego oraz skutecznosc jego leczenia $u$ doroslych mieszkancow naszego kraju. Wyniki programu WOBASZ. Kardiol. Pol. 2005; 63 (supl. 4): S614-619 (in Polish).

[3] $2013 \mathrm{ESH} / \mathrm{ESC}$ Guidelines for the management of arterial hypertension, Journal of Hypertension 2013, 31:1281-1357

[4] Lurbe E, Cifkova R, Cruickshank JK, et al. Management of high blood pressure in children and adolescents: recommendations of the European Society of Hypertension. J Hypertens 2009; 27:1719-1742

[5] 5. Scherer PE, Williams S, Fogliano M, et al. A novel serum protein similar to Clq, produced exclusively in adipocytes. J Biol Chem. 1995; 270:26746-26749

[6] Franklin SS, Gustin WIV, Wong ND, et al. Haemodynamic patterns of age-related changes in blood pressure. The Framingham Heart Study. Circulation 1997; 96:308-315.

[7] Stamler J. The INTERSALT Study: background, methods, findings, and implications. Am J Clin Nutr February 1997 vol. 65 no. 2 626S-642S

[8] Nadcisnienie tetnicze u osob w wieku podeszlym. (red)T. Grodzicki, J.Kocemba, B. Gryglewska (in Polish)

[9] B. Sumathi, Dr. A. Santhakumaran Pre-Diagnosis of Hypertension Using Artificial Neural Network

[10] Kaur A., Bhardwaj A. Artificial Intelligence in Hypertension Diagnosis: A Review. International Journal of Computer Science and Information Technologies, Vol. 5 (2) , 2014, 2633-2635

[11] Samant, Rahul, and Srikantha Rao. Evaluation of Artificial Neural Networks in Prediction of Essential Hypertension. International Journal of Computer Applications 2013

[12] Shehu, N., S. U. Gulumbe, and H. M. Liman. Comparative study between conventional statistical methods and neural networks in predicting hypertension status. Advances in Agriculture, Sciences and Engineering Research 2013

[13] Ture, Mevlut, et al. Comparing classification techniques for predicting essential hypertension. Expert Systems with Applications, Elsevier 2005

[14] Djam, X. Y., and Y. H. Kimbi. Fuzzy expert system for the management of hypertension. The Pacific Journal of Science and Technology 2011.

[15] Zurada, J. M. Introduction to artificial neural systems. West Publishing Co. 1992.

[16] Nawarycz T., Pytel K., Gazicki-Lipman M., Drygas W., OstrowskaNawarycz L., A Fuzzy Logic Approach to The Evaluation of Health Risks Associated with Obesity. FedCSIS 2013: 231-234 